\title{
A non-small cell lung cancer (NSCLC) patient with leptomeningeal metastasis harboring rare epidermal growth factor receptor (EGFR) mutations G719S and L861Q benefited from doubling dosage of osimertinib: a case report
}

\author{
Chang-Guo Shan ${ }^{1 \#}$, Hui Wang ${ }^{1 \#}$, Tao Lin ${ }^{2}$, Da Liu ${ }^{2}$, Lei Wen ${ }^{1}$, Zhi-Jie Chen ${ }^{2}$, Jun-Jie Zhen ${ }^{1}$, \\ Ming-Yao Lai ${ }^{1}$, Lu Zhang ${ }^{3}$, Xiao Zou ${ }^{3}$, Wei-Ping Hong ${ }^{1}$, Lin-Bo Cai ${ }^{1} \wedge$ \\ ${ }^{1}$ Department of Neuro-Oncology, Guangdong Sanjiu Brain Hospital, Guangzhou, China; ${ }^{2}$ Department 2 of Neurosurgery, Guangdong Sanjiu Brain \\ Hospital, Guangzhou, China; ${ }^{3}$ Burning Rock Biotech, Guangzhou, China \\ \#These authors contributed equally to this work. \\ Correspondence to: Dr. Wei-Ping Hong. Department of Oncology, Guangdong Sanjiu Brain Hospital, No. 578 Shatai South Road, Baiyun District, \\ Guangzhou 510510, China. Email: 53874279@qq.com; Dr. Lin-Bo Cai. Department of Oncology, Guangdong Sanjiu Brain Hospital, No. 578 Shatai \\ South Road, Baiyun District, Guangzhou 510510, China. Email: cailinbo999@163.com.
}

\begin{abstract}
Leptomeningeal metastasis $(\mathrm{LM})$ is a rare but lethal complication of advanced non-small cell lung cancer (NSCLC) that has a devastating impact on patient survival and quality of life. Osimertinib, an irreversible tyrosine kinase inhibitor, is approved as a therapy for advanced NSCLC with epidermal growth factor receptor (EGFR) mutation. However, the efficacy and optimal dosage of osimertinib in the treatment of NSCLC patients with LM who harbor uncommon EGFR mutations have yet to be fully investigated. Herein, we report a case of an advanced NSCLC patient with LM carrying EGFR G719S and L861Q, who was successfully treated by osimertinib at $160 \mathrm{mg}$. The patient initially presented with clear cell renal carcinoma and renal metastatic adenocarcinoma, and underwent right nephrectomy. At 2 months after nephrectomy, he developed a disturbance of consciousness and was subsequently diagnosed with NSCLC with LM by meningeal biopsy pathology and cerebrospinal fluid (CSF) cytology. Next-generation sequencing detected the rare EGFR mutations G719S and L861R in the meningeal biopsy tissues. The patient was then administered osimertinib at $80 \mathrm{mg}$ quaque die (QD); after 1 month of treatment, his symptoms were alleviated. However, two months later, he experienced epileptic episode. Subsequently, the osimertinib dosage was doubled to $160 \mathrm{mg}$ QD. After 1 month of treatment, the patient achieved central nervous system (CNS) response, and at the time of this manuscript's submission, he had maintained stable disease (SD) for more than 1 year. To our knowledge, this study provides the first clinical evidence that the administration of osimertinib at $160 \mathrm{mg}$ once daily can achieve an encouraging, durable response in an NSCLC patient with LM carrying EGFR G719S and L861Q.
\end{abstract}

Keywords: Case report; leptomeningeal metastasis (LM); non-small cell lung cancer (NSCLC); tyrosine kinase inhibitor; osimeritinib; rare epidermal growth factor receptor mutations (rare EGFR mutations)

Submitted Sep 24, 2020. Accepted for publication Apr 23, 2021.

doi: 10.21037/apm-20-2556

View this article at: http://dx.doi.org/10.21037/apm-20-2556

^ ORCID: 0000-0003-1011-9868. 


\section{Introduction}

Leptomeningeal metastasis (LM) occurs in $3-4 \%$ of advanced non-small cell lung cancer (NSCLC) patients $(1,2)$. Among NSCLC patients with epidermal growth factor receptor (EGFR) mutations, the incidence rate of $\mathrm{LM}$ has been reported to be as high as $9 \%(1,3)$. The median overall survival (OS) for NSCLC patients with LM ranges from 3-10 months $(1,4)$. Surgery, whole-brain radiotherapy (WBRT), and intrathecal chemotherapy are the traditional treatment approaches for LM. However, due to the location of lesions, many patients are unable to undergo surgery, while WBRT and chemotherapy are associated with significant adverse effects, which limit their clinical utility considerably (5-7). Recently, targeted therapies have shown enormous potential in the treatment of NSCLC patients with LM.

In the setting of LM, drug delivery is compounded by the blood-brain barrier (BBB), which reduces the efficacy of a number of established tyrosine kinase inhibitors (TKIs). Osimertinib, an oral, third-generation TKI with the ability to penetrate the BBB (8), was first approved for the treatment of metastatic NSCLC patients with epidermal growth factor receptor $(E G F R)$ p. Thr790Met point mutation (T790M) who show disease progression after receiving first or second-generation EGFR-TKIs. In the recently updated FLAURA phase 3 study of 556 previously untreated NSCLC patients harboring EGFR exon 19 deletion or L858R mutation, osimertinib showed superiority over the standard EGFR-TKIs gefitinib or erlotinib in median overall survival (38.6 vs. 31.8 months) and a similar safety profile (adverse events of grade 3 or higher $42 \%$ vs. 47\%) (9). Subsequently, it was also approved as a firstline treatment for metastatic NSCLC patients harboring the EGFR exon 19 deletion or L858R mutations. A recent phase I trial of osimertinib showed an encouraging (central nervous system) CNS response in NSCLC patients with LM harboring EGFR exon 19 deletions or L858R mutations, with or without the presence of EGFR T790M; the median progression-free survival (PFS) and overall survival (OS) of these patients were 8.6 months and 11.1 months, respectively (10). However, the efficacy and optimal dosage of osimertinib for treating patients with NSCLCinduced LM who carry uncommon EGFR mutations have not been determined. Here, we present the management of a patient with two primary cancers and leptomeningeal metastases. An LM biopsy was obtained incidentally and enabled pathologic identification of thet tissue of origin.
Furthermore, molecular testing of the biopsy detected two rare EGFR mutations G719S and L861Q, which was treated with osimertinib at $160 \mathrm{mg}$ q.d. and lead to a PFS of 12 months as of the time of manuscript preparation. To our knowledge, this report is the first to provide clinical evidence of durable response to osimertinib at $160 \mathrm{mg}$ q.d. in an NSCLC patient with LM that harbored EGFR G719S and L861Q mutations. We present the following article in accordance with the CARE reporting checklist (available at http://dx.doi.org/10.21037/apm-20-2556).

\section{Case presentation}

A 44-year-old smoking man underwent surgical resection for right renal mass in April, 2019. Pathology of resected renal tumor revealed components of both clear cell renal cell carcinoma and metastatic adenocarcinoma, indicating synchronous bi-primary cancer of clear cell renal cell carcinoma and adenocarcinoma of unknown primary site. Further PET/CT suggested potential bone metastasis, lung, and increased metabolism in the bilateral occipital lobes. The patient's treatment history is shown in Figure 1.

In June 2019, the patient presented with behavioral abnormalities, headaches, and unconsciousness, combined with intracranial hypertension. His Karnofsky performance score (KPS) was 20. He displayed no fever, cough, or dyspnea. Magnetic resonance imaging (MRI) showed meningeal enhancement (Figure 2A). Atypical cells were observed in the patient's cerebrospinal fluid (CSF), and his carcinoembryonic antigen (CEA) levels were abnormal, measuring $14.53 \mathrm{ng} / \mathrm{mL}$. Subsequently, the patient was diagnosed with $\mathrm{LM}$.

During external brain ventricular drainage surgery to relieve the intracranial hypertension, a neoplasm was found and biopsied from the surface of the meninges. Hematoxylin and eosin staining of the brain lesion revealed that epithelioid tumor cells with a glandular tubular arrangement had infiltrated the cerebral cortex and the surface of the intracranial dura. Furthermore, immunohistochemistry (IHC) revealed CK(+), CK7(+), TTF-1(+), and Napsin-a(+), which indicated that the LM had originated from lung adenocarcinoma. Molecular profiling of both renal tissue, collected during nephrectomy in April, 2019, and the meninges lesion was performed. Two rare EGFR mutations G719S and L861Q were detected in the meninges lesion; however, no EGFR mutation was detected in the renal lesion, which further suggested that the LM was derived from the lung tumor. 


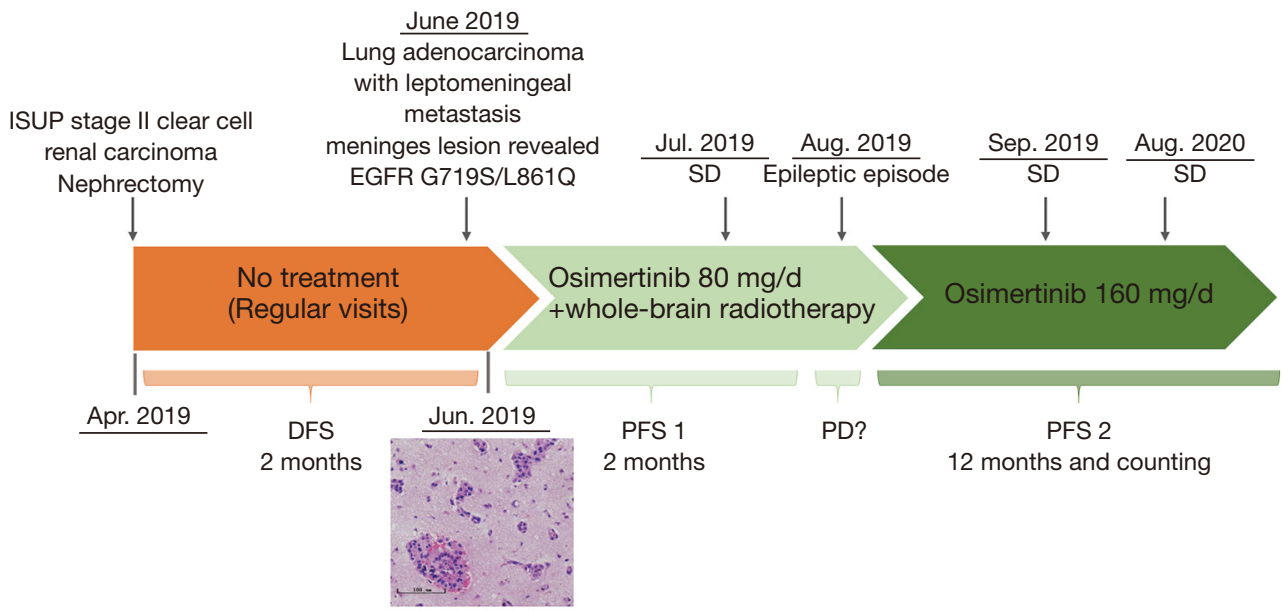

Figure 1 A summary of the patient's treatment history. PD, progressive disease; PFS, progression-free survival; NGS, next-generation sequencing; SD, stable disease. A meningeal lesion was found in Jun 2019 and hemotaxylin and eosin staining $(\times 100)$ results showed leptomeningeal metastatic adenocarcinoma.

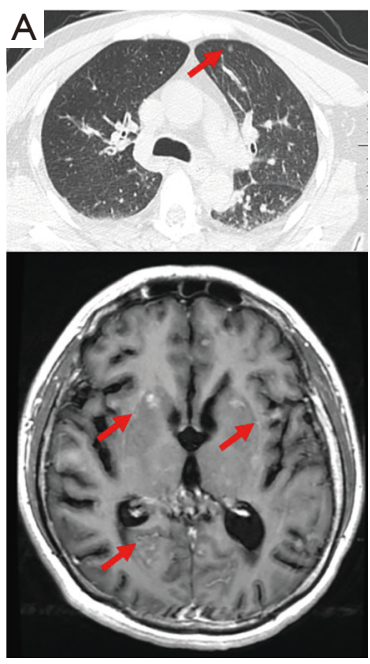

Before osimertinib
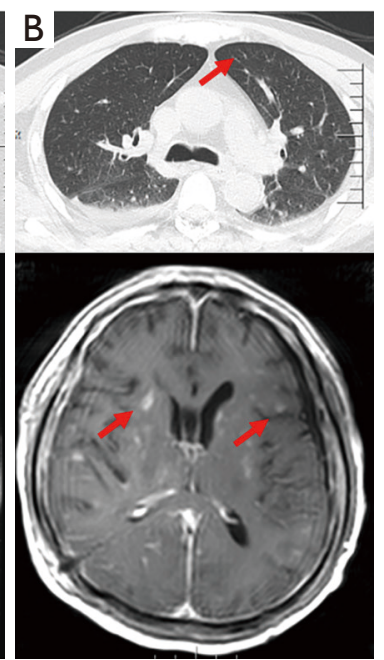

1 month after initiation of Osimertinib 80 QD+WBRT (SD)
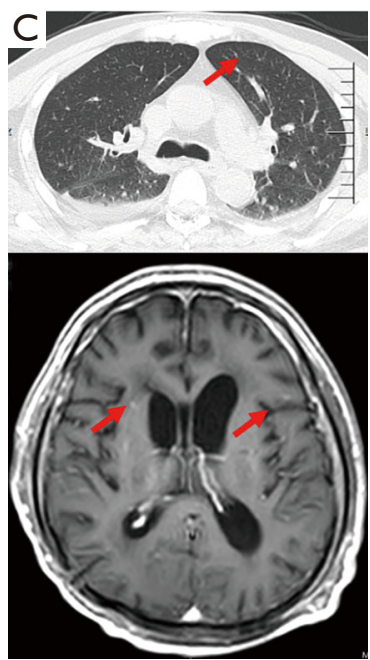

3 months after initiation of osimertinib 160mg QD (SD)
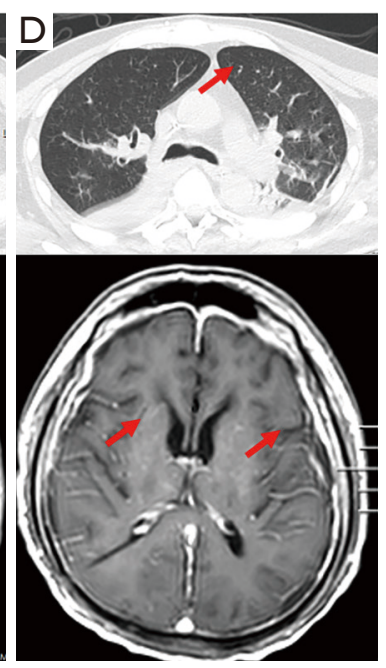

12 months after initiation of osimertinib 160mg QD (SD)

Figure 2 Computed tomography (CT) scans and magnetic resonance imaging (MRI) of the patient's lung and brain at treatment milestones. (A) Before the initiation of osimertinib. (B) After 1 month of osimertinib $80 \mathrm{mg}$ QD. (C) After 3 months of osimertinib $160 \mathrm{mg}$ QD. (D) Resolution of pulmonary nodules (arrows, upper panel) and continuous improvement in meningeal enhancement (arrows, lower panel) were observed after 12 months of treatment with osimertinib $160 \mathrm{mg}$ QD.

Accordingly, the patient was administered with osimertinib (80 mg QD) combined with WBRT (36 Gy in 18 fractions over 3 weeks) in June and July, 2019. Despite a reduction in meningeal enhancement and shrinkage of the pulmonary nodules (Figure 2B), in August, the patient experienced an epileptic seizure and subsequently appeared coma. Consequently, the dose of osimertinib was doubled (160 mg QD) with the consent of family members, and the patient regained consciousness 2 weeks later. The main adverse events were diarrhea and skin rash, which 
were grade 2. Reexamination in November, 2019 showed considerable shrinkage of the pulmonary nodules and less meningeal enhancement (Figure 2C). In September 2020, the pulmonary nodules were undetectable, which was accompanied by a continuous reduction in meningeal enhancement (Figure 2D). At the time this manuscript was submitted, the patient had achieved stable disease (SD), with a PFS of 12 months and counting.

All procedures performed in studies involving human participants were in accordance with the ethical standards of the institutional and/or national research committee(s) and with the Helsinki Declaration (as revised in 2013). Written informed consent was obtained from the patient.

\section{Discussion}

This is a rare case of a patient who initially presented with synchronous bi-primary cancer of clear cell renal carcinoma and renal metastatic adenocarcinoma, and was subsequently presented with leptomeningeal metastases. Therefore, where possible, it is advisable to perform meningeal biopsy or obtain CSF for identification purposes in similar cases. At the same time, it also reminds us that patients with critical LM like this case need multidisciplinary treatments to figure out condition and relieve symptoms.

An important point to note from this case is that the dosage of osimertinib was twice of the regular dose. As the patient was deemed to be too weak to undergo radiotherapy or chemotherapy, targeted therapy was chosen. Based on the two rare EGFR mutations detected in the meningeal biopsy specimen, G719s (exon 18) and L861Q (exon 21), osimertinib $80 \mathrm{mg}$ daily was administered initially. However, despite showing an initial response, after 2 months, the patient experienced an epileptic fit and disorder of consciousness, which improved after the standard dosage of osimertinib was doubled.

Recently, the clinical activity of osimertinib in NSCLC patients with rare EGFR mutations has been reported. In a single-arm phase II trial, osimertinib $80 \mathrm{mg}$ daily achieved a median PFS and an objective response rate (ORR) of 8.2 months and $50 \%$, respectively, in the treatment of NSCLC patients harboring uncommon mutations (11). In the phase 1 BLOOM study, osimertinib at $160 \mathrm{mg}$ QD showed a CNS response and a manageable safety profile in patients with EGFRm NSCLC and LM, regardless of EGFR T790M status, achieving a median PFS and OS of 8.6 and 11.1 months, respectively (10). As described in this case report, a higher than standard dose of osimertinib exhibited an encouraging response, with PFS more than 12 months in a NSCLC patient with EGFR G719s and L861Q mutations who, at a dosage of $80 \mathrm{mg}$ daily, achieved a CNS response for only 2 months. Our case provides further clinical evidence for the administration of osimertinib at $160 \mathrm{mg}$ QD for treating NSCLC patients with LM harboring EGFR G719S and L861Q mutations. In addition to osimertinib, there is evidence that a novel third-generation EGFR inhibitor TAS-121 inhibited G719X and L861Q mutations as potently as osimertinib and displayed antitumor activity in EGFR G719S-mutant cell models (12). An initial phase 1 study in Japanese patients showed that TAS-121 was well tolerated and had antitumor activity in T790M-positive advanced NSCLC patients, although more clinical trials are warranted for further characterization.

In conclusion, osimertinib treatment at $160 \mathrm{mg}$ QD achieved a durable clinical response in a patient with NSCLC and LM harboring rare EGFR mutations G719s and L861Q, who initially presented with clear cell renal carcinoma and renal metastatic adenocarcinoma.

\section{Acknowledgments}

We thank to the patient and his family. We thank the staff in the Department of Oncology at Guangdong 999 Brain Hospital.

Funding: None.

\section{Footnote}

Reporting Checklist: The authors have completed the CARE reporting checklist. Available at http://dx.doi.org/10.21037/ apm-20-2556

Conflicts of Interest: All authors have completed the ICMJE uniform disclosure form (available at http://dx.doi. org/10.21037/apm-20-2556). LZ and XZ report personal fees from Burning Rock Biotech during the conduct of the study. The other authors have no conflicts of interest to declare.

Ethical Statement: The authors are accountable for all aspects of the work in ensuring that questions related to the accuracy or integrity of any part of the work are appropriately investigated and resolved. All procedures performed in studies involving human participants were in accordance with the ethical standards of the institutional 
and/or national research committee(s) and with the Helsinki Declaration (as revised in 2013). Written informed consent was obtained from the patient.

Open Access Statement: This is an Open Access article distributed in accordance with the Creative Commons Attribution-NonCommercial-NoDerivs 4.0 International License (CC BY-NC-ND 4.0), which permits the noncommercial replication and distribution of the article with the strict proviso that no changes or edits are made and the original work is properly cited (including links to both the formal publication through the relevant DOI and the license). See: https://creativecommons.org/licenses/by-nc-nd/4.0/.

\section{References}

1. Li YS, Jiang BY, Yang JJ, et al. Leptomeningeal Metastases in Patients with NSCLC with EGFR Mutations. J Thorac Oncol 2016;11:1962-9.

2. Liao BC, Lee JH, Lin CC, et al. Epidermal Growth Factor Receptor Tyrosine Kinase Inhibitors for NonSmall-Cell Lung Cancer Patients with Leptomeningeal Carcinomatosis. J Thorac Oncol 2015;10:1754-61.

3. Kuiper JL, Hendriks LE, van der Wekken AJ, et al. Treatment and survival of patients with EGFR-mutated non-small cell lung cancer and leptomeningeal metastasis: A retrospective cohort analysis. Lung Cancer 2015;89:255-61.

4. Xu Y, Hu M, Zhang M, et al. Prospective study revealed prognostic significance of responses in leptomeningeal metastasis and clinical value of cerebrospinal fluid-based liquid biopsy. Lung Cancer 2018;125:142-9.

5. Morris PG, Reiner AS, Szenberg OR, et al.

Cite this article as: Shan CG, Wang H, Lin T, Liu D, Wei L, Chen ZJ, Zhen JJ, Lai MY, Zhang L, Zou X, Hong WP, Cai LB. A non-small cell lung cancer (NSCLC) patient with leptomeningeal metastasis harboring rare epidermal growth factor receptor (EGFR) mutations G719S and L861Q benefited from doubling dosage of osimertinib: a case report. Ann Palliat Med 2021;10(5):5897-5901. doi: 10.21037/apm-20-2556
Leptomeningeal metastasis from non-small cell lung cancer: survival and the impact of whole brain radiotherapy. J Thorac Oncol 2012;7:382-5.

6. Cheng H, Perez-Soler R. Leptomeningeal metastases in non-small-cell lung cancer. Lancet Oncol 2018;19:e43-e55.

7. Chamberlain MC. Leptomeningeal metastasis. Curr Opin Oncol 2010;22:627-35.

8. Ballard P, Yates JW, Yang Z, et al. Preclinical Comparison of Osimertinib with Other EGFR-TKIs in EGFR-Mutant NSCLC Brain Metastases Models, and Early Evidence of Clinical Brain Metastases Activity. Clin Cancer Res 2016;22:5130-40.

9. Ramalingam SS, Vansteenkiste J, Planchard D, et al. Overall Survival with Osimertinib in Untreated, EGFR-Mutated Advanced NSCLC. N Engl J Med 2020;382:41-50.

10. Yang JCH, Kim SW, Kim DW, et al. Osimertinib in Patients With Epidermal Growth Factor Receptor Mutation-Positive Non-Small-Cell Lung Cancer and Leptomeningeal Metastases: The BLOOM Study. J Clin Oncol 2020;38:538-47.

11. Cho JH, Lim SH, An HJ, et al. Osimertinib for Patients With Non-Small-Cell Lung Cancer Harboring Uncommon EGFR Mutations: A Multicenter, OpenLabel, Phase II Trial (KCSG-LU15-09). J Clin Oncol 2020;38:488-95.

12. Ito K, Nishio M, Kato M, et al. TAS-121, A Selective Mutant EGFR Inhibitor, Shows Activity Against Tumors Expressing Various EGFR Mutations Including T790M and Uncommon Mutations G719X. Mol Cancer Ther 2019;18:920-8.

(English Language Editor: J. Reynolds) 